\title{
Proximity of Residence to Bodies of Water and Risk for West Nile Virus Infection: A Case-Control Study in Houston, Texas
}

\author{
Melissa S. Nolan, ${ }^{1}$ Ana Zangeneh, ${ }^{1}$ Salma A. Khuwaja, ${ }^{2}$ Diana Martinez, ${ }^{3}$ \\ Susan N. Rossmann, ${ }^{4}$ Victor Cardenas, ${ }^{1}$ and Kristy O. Murray ${ }^{1}$ \\ ${ }^{1}$ Center for Infectious Diseases, School of Public Health, The University of Texas Health Science Center at Houston, \\ Houston, TX 77030, USA \\ ${ }^{2}$ Office of Surveillance and Public Health Preparedness, Houston Department of Health and Human Services, \\ Houston, TX 77054, USA \\ ${ }^{3}$ Office of Disease Control and Clinical Prevention, Harris County Public Health \& Environmental Services, \\ Houston, TX 77027, USA \\ ${ }^{4}$ Gulf Coast Regional Blood Center, Houston, TX 77054, USA
}

Correspondence should be addressed to Kristy O. Murray, kristy.o.murray@uth.tmc.edu

Received 1 August 2011; Revised 27 September 2011; Accepted 19 October 2011

Academic Editor: Roy A. Hall

Copyright ( $) 2012$ Melissa S. Nolan et al. This is an open access article distributed under the Creative Commons Attribution License, which permits unrestricted use, distribution, and reproduction in any medium, provided the original work is properly cited.

West Nile virus (WNV), a mosquito-borne virus, has clinically affected hundreds of residents in the Houston metropolitan area since its introduction in 2002. This study aimed to determine if living within close proximity to a water source increases one's odds of infection with WNV. We identified 356 eligible WNV-positive cases and 356 controls using a population proportionate to size model with US Census Bureau data. We found that living near slow moving water sources was statistically associated with increased odds for human infection, while living near moderate moving water systems was associated with decreased odds for human infection. Living near bayous lined with vegetation as opposed to concrete also showed increased risk of infection. The habitats of slow moving and vegetation lined water sources appear to favor the mosquito-human transmission cycle. These methods can be used by resource-limited health entities to identify high-risk areas for arboviral disease surveillance and efficient mosquito management initiatives.

\section{Introduction}

Houston, Texas, is a metropolis in the southeastern United States with around four million residents [1]. West Nile virus (WNV) human cases were first reported locally in 2002 [2] and have since become endemic with human cases reported annually [3]. WNV is an arboviral disease from the Flaviviridae family whose main transmission cycle occurs between birds and mosquitoes; humans serve as an incidental host. In southeastern United States, Culex quinquefasciatus mosquitoes have been demonstrated as important vectors of WNV disease transmission $[2,4]$.

In the United States, WNV transmission season traditionally occurs from spring to fall, with a peak in late summer [2]. In warm weather, mosquito larval development occurs within days $[5,6]$ allowing for rapid reproduction of new mosquito populations. Mosquito larval development occurs in water bodies with each species having their own preferential type. Culex quinquefasciatus mosquitoes have a diverse larval habitat range, with high larval counts near human habitation [7, 8]. Mosquito control efforts in Houston, Texas, target residential areas where either mosquito pools or dead birds are positive for WNV disease. Targeted areas are identified through random mosquito trapping and reporting of dead birds by residents. The ecological dynamic between vector, reservoir, and human habitats is critical to understand when examining risk for human WNV infection. While this vector's larval habitat preferences are known, no studies to date have examined direct associations between larval water habitats and WNV human disease transmission. This paper presents a novel method for examining disease clustering and its spatial association with water sources. 


\section{Methods}

A case-control study design was used to determine the association between water sources and the risk of human infection with WNV.

2.1. Case Selection. Cases were defined as WNV-positive patients identified through local surveillance performed by the Houston Department of Health and Human Services (HDHHS), Harris County Public Health and Environmental Services (HCPHES), or the Gulf Coast Regional Blood Center (GCRBC). Local surveillance identified cases either by state mandatory reporting laws or by national blood donation testing guidelines that required laboratory confirmation of WNV human disease. Previous research has shown that the highest rates of WNV human seroprevalence were among those who reported a history of being outside during the hours of dusk and dawn [9]. These hours are concurrent with the peak activity time of Culex quinquefasciatus mosquitoes. Since most people are at home during dusk and dawn, it was resolved that cases are most likely exposed while at home. It was determined appropriate to use cases' home address at time of disease development as their location of mosquito exposure. Cases' home addresses were collected via case investigations performed by HDHHS, HCPHES, or GCRBC during 2002 and 2009. Exclusion criteria included evidence of nonlocally acquired disease as documented in the case investigation nonrecognition of address by MapMarker USA version 14 geocoding software, or home address falling outside the metropolitan's geographic area as determined by the geocoding software. After applying the exclusion criteria, we had 356 residential addresses from cases for final analysis.

2.2. Control Selection. Controls were defined as selected block centroids generated from the United States Census Bureau decennial data (http://www.census.gov/). Controls were selected using two methods: a population proportionate to size sampling method which takes into account varying population densities within the metropolitan city and a random sampling method. There were three selection frames that were used to identify the final control. In descending order the frames were census tract level, block group, and finally block. The population proportionate to size sampling methods was used to select the initial frame: census tract level. It was understood that population distribution was uniform throughout the census tracts selected; therefore, we used a random selection method for the two additional frames: block group and block. Since the smallest defined census level is a block, the centroid of the block level was used as a surrogate for control households. Based on sample size calculations, a 1:1 case-control ratio was determined appropriate to satisfy statistical significance using discipline standards; therefore, 356 control addresses were selected for final analysis.

2.3. Data Analysis. Spatial analysis of case and control residential distances' to local water body sources was performed using MapInfo v9.5.1 software. Shapefiles of water sources within the metropolitan's geographic parameters were provided in kind by Dr. Irina Cech, professor at the University of Texas Health Science Center at Houston. The shapefiles were based on United States Geological Survey water source definitions and data. Case and control residential coordinates were superimposed onto the water source shapefile. Water source labels were used to identify the particular water source, that is, Cedar Spring, Lou River, Brays Bayou, and so forth. The water source type was inferred from these labels. Using the software's measurement tool, we measured the distance from each case/control point, to the closest water source, excluding salt water sources since Culex quinquefasciatus mosquitoes do not utilize salt water sources as larval habitats [5]. For each case/control point we recorded the proximity to the closest water source, the type of the particular water source, and the name of the particular water source. We used STATA v11.0 (College Station, Texas) to run all statistical analyses. Chi-squared tables and logistic regression were used to analyze the significance of proximity to a water source between the two populations. Odds ratios, $95 \%$ confidence intervals (CIs), and $P$ values were computed to analyze the significance of three factors: specified residential proximity to a water source; proximity to a particular water source type; proximity to a particular water source. Attack rates (number of WNV human cases over total number of households) were calculated for each census tract and mapped to spatially identify areas of high WNV human transmission. A Getis Ord hot spot analysis was performed using ESRI ArcGIS 10.0 to determine concentrations of high and low human disease clustering. The GetisOrd (Gi) hot spot analysis identifies clusters of higher and lower magnitude than would be randomly found and statistical output is in the form of a $Z$ score known as a GiZ score. Areas of high clustering were indicated by a GiZ score of 1.96 or greater, and areas of low clustering were indicated by a GiZ score of -1.96 or less.

\section{Results}

On average, cases and controls resided the same proximity from water sources $\left[x_{0}\right.$ (controls) $=892$ meters, $x_{1}$ (cases) $=$ 931 meters]. Using linear regression, we found no statistical association between residential proximity to water and odds for human WNV infection. However, when we binomiallycoded at varying distances ranging from 50 to 750 meters, we found a significant protective trend from distances ranging from 50 to 200 meters (Table 1). Living less than or equal to 200 meters from a water source $\left(x^{2}=6.67, P<0.01\right)$ was found to be protective from infection by a factor of 0.54 .

Water source types were analyzed for association with odds for human WNV infection using odds ratios and chisquared tests, as seen in Table 2. We examined the six most common water source types. Two water source types were statistically associated with odds of human infection. Living near a creek increased one's odds of human infection by a factor of $1.37(P=0.09)$. Living near a spring decreased one's odds of human infection by a factor of $0.55(P=0.06)$. To further analyze these associations, we created two groupings 
TABLE 1: Distance of case residence compared to US Census control centroids to water source in meters, evaluated by odds ratio (OR), $95 \%$ confidence intervals $(\mathrm{CI})$, and significance ( $P$ value).

\begin{tabular}{lccc}
\hline Distance $(\mathrm{m})$ & OR & $95 \%$ CI & $P$ value \\
\hline 50 & 0.10 & $(0.01,0.42)$ & $<0.01$ \\
100 & 0.21 & $(0.07,0.42)$ & $<0.01$ \\
150 & 0.35 & $(0.18,0.66)$ & $<0.01$ \\
200 & 0.54 & $(0.32,0.89)$ & 0.01 \\
250 & 0.70 & $(0.46,1.05)$ & 0.07 \\
300 & 0.76 & $(0.52,1.11)$ & 0.14 \\
350 & 0.78 & $(0.54,1.12)$ & 0.16 \\
400 & 0.82 & $(0.58,1.16)$ & 0.24 \\
450 & 0.82 & $(0.59,1.14)$ & 0.22 \\
500 & 0.85 & $(0.62,1.17)$ & 0.31 \\
550 & 0.87 & $(0.63,1.19)$ & 0.35 \\
600 & 0.78 & $(0.57,1.07)$ & 0.11 \\
650 & 0.84 & $(0.62,1.15)$ & 0.26 \\
700 & 0.89 & $(0.66,1.21)$ & 0.45 \\
750 & 0.92 & $(0.68,1.25)$ & 0.60 \\
\hline
\end{tabular}

based on slow moving and moderate moving water source types. A grouping of slow moving water bodies (creeks and gullies) was found to increase one's odds of human infection by a factor of $1.45(P=0.03)$. A grouping of narrow moderate moving water bodies (streams and rivers) was found to be protective against human infection by a factor of $0.50(P=0.02)$.

Particular water sources were evaluated for association with odds for human WNV infection by odds ratios and chisquared tests, as seen in Table 3 . The eleven most common specific water sources were analyzed. Two water body sources were significantly associated with increased odds for human infection. Living close to White Oak Bayou $(P=0.01)$ increased one's odds of human infection by a factor of 2.25. Additionally, living near Cypress Creek $(P=0.02)$ was also associated with increased odds of human infection by a factor of 2.54. Since Cypress Creek has several tributaries, an additional category was made that included all feeders for Cypress Creek. This group had the strongest significance of all water bodies $(P<0.01)$ with increased odds of human infection by a factor of 1.93. We also found that living close to Buffalo Bayou had increased odds of human infection by a factor of 1.59 , which neared significance $(P=0.07)$.

Spatial distribution of WNV attack rates per 10,000 population by census tract illustrates that the highest risk area of transmission is in Northwest Houston as seen in Figure 1. Hot spot analysis confirmed that there were significant clusters of cases in Houston as seen in Figure 2. The areas of highest valued clusters were along the Northwest corner of Harris County, which overlaps Cypress Creek and its feeders. Figure 3 demonstrates the spatial relevance of the Houston area inlaid within Harris County, in relation to the state of Texas, and the United States of America.
TABle 2: Proximity of residence to water source types in cases versus controls, evaluated by odds ratio (OR), 95\% confidence intervals $(\mathrm{CI})$, and significance ( $P$ value).

\begin{tabular}{lccc}
\hline Water source type* & OR & $95 \%$ CI & $P$ value \\
\hline Bayou & 1.15 & $(0.84,1.56)$ & 0.36 \\
Creek & 1.37 & $(0.93,2.02)$ & 0.09 \\
Ditch & 0.49 & $(0.13,1.60)$ & 0.19 \\
Gully & 1.50 & $(0.73,3.16)$ & 0.23 \\
Lake & 1.50 & $(0.73,3.16)$ & 0.23 \\
Stream & 0.55 & $(0.27,1.08)$ & 0.06 \\
Creek and gully & 1.45 & $(1.02,2.07)$ & 0.03 \\
Stream and river & 0.50 & $(0.25,0.95)$ & 0.02 \\
\hline
\end{tabular}

* As defined by the United States Geological Survey.

TABle 3: Proximity of residence to particular water sources in cases versus controls, evaluated by odds ratio (OR), 95\% confidence intervals $(\mathrm{CI})$, and significance ( $P$ value).

\begin{tabular}{lccc}
\hline Particular water source & OR & $95 \%$ CI & $P$ value \\
\hline Bering Ditch & 0.66 & $(0.14,2.82)$ & 0.52 \\
Berry Bayou & 1.00 & $(0.26,3.78)$ & 1.00 \\
Brays Bayou & 0.73 & $(0.43,1.23)$ & 0.21 \\
Buffalo Bayou & 1.59 & $(0.93,2.75)$ & 0.07 \\
Cypress Creek & 2.54 & $(1.10,6.35)$ & 0.02 \\
Cypress Creek and tributaries & 1.93 & $(1.14,3.33)$ & 0.01 \\
Greens Bayou & 0.66 & $(0.26,1.59)$ & 0.31 \\
Halls Bayou & 1.00 & $(0.40,2.47)$ & 1.00 \\
Hunting Bayou & 1.89 & $(0.69,5.66)$ & 0.17 \\
Little White Oak Bayou & 1.81 & $(0.74,4.72)$ & 0.15 \\
Sims Bayou & 0.57 & $(0.19,1.61)$ & 0.25 \\
White Oak Bayou & 2.25 & $(1.15,4.55)$ & 0.01 \\
\hline
\end{tabular}

\section{Discussion}

This is the first known case-control study to perform a spatial analysis of human WNV infection risk with regard to proximity of residences to water sources serving as surrogates for potential aquatic larval habitats. Overall, we found no direct association between proximity of residences to water sources and odds of WNV human infection in Houston, Texas. However, we found a significant trend of decreased risk of infection among people living within 200 meters of a water source. It is conjectured that areas closest to water sources are the primary target of mosquito control programs, therefore decreasing the risk of transmission at closer distances. We did find a pattern of increasing odds ratios as distance increased by 50 -meter intervals, suggesting that mosquitoes in Houston have an expansive flight range that is important in the ecology of disease transmission. Culex quinquefasciatus mosquitoes are known to have an expansive flight range with recapture documented up to 1000 meters outside of their release site [10]. One speculation could be that the use of adulticides along water bodies could temporarily suspend adult mosquito activity allowing for higher mosquito activity occurring at greater distances. Although adulticides are the primary mosquito 


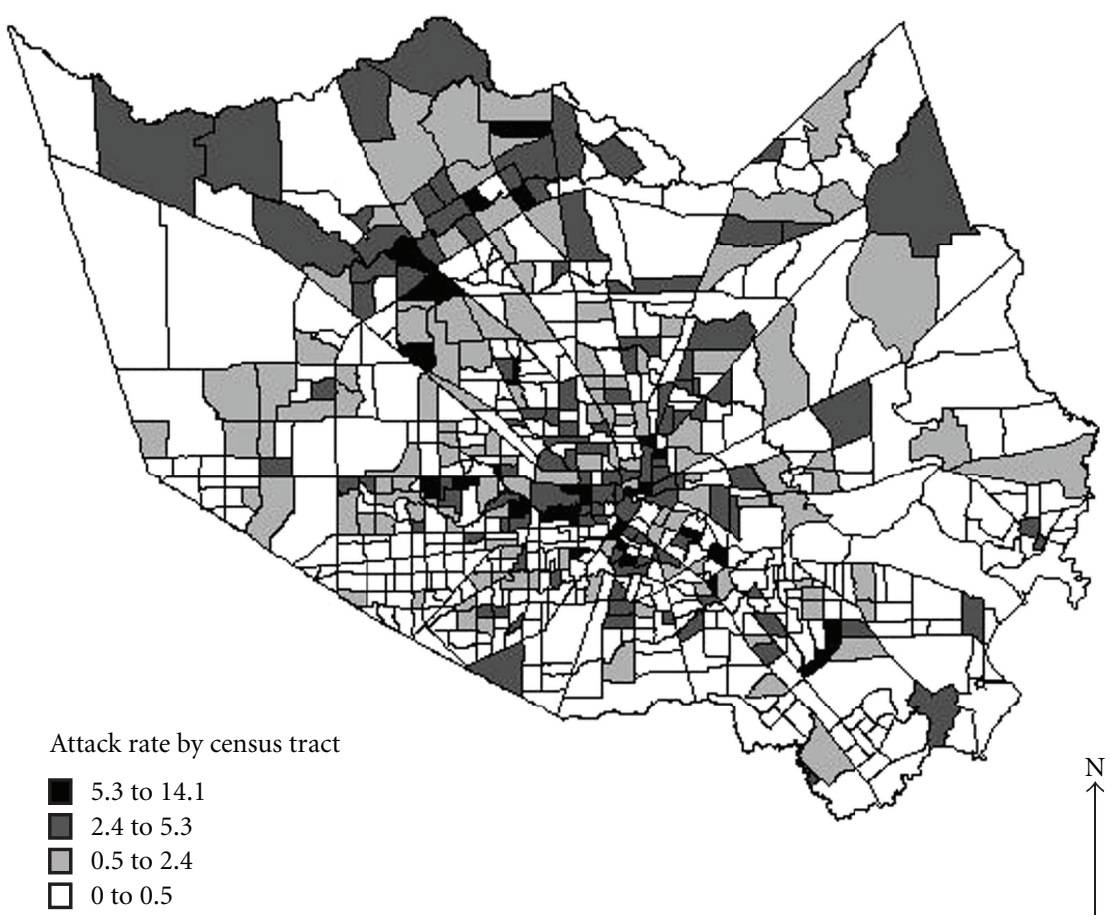

Figure 1: Attack rate: number of reported West Nile virus cases per 10,000 population using 2000 US census tract data in the Houston metropolitan area, Texas.

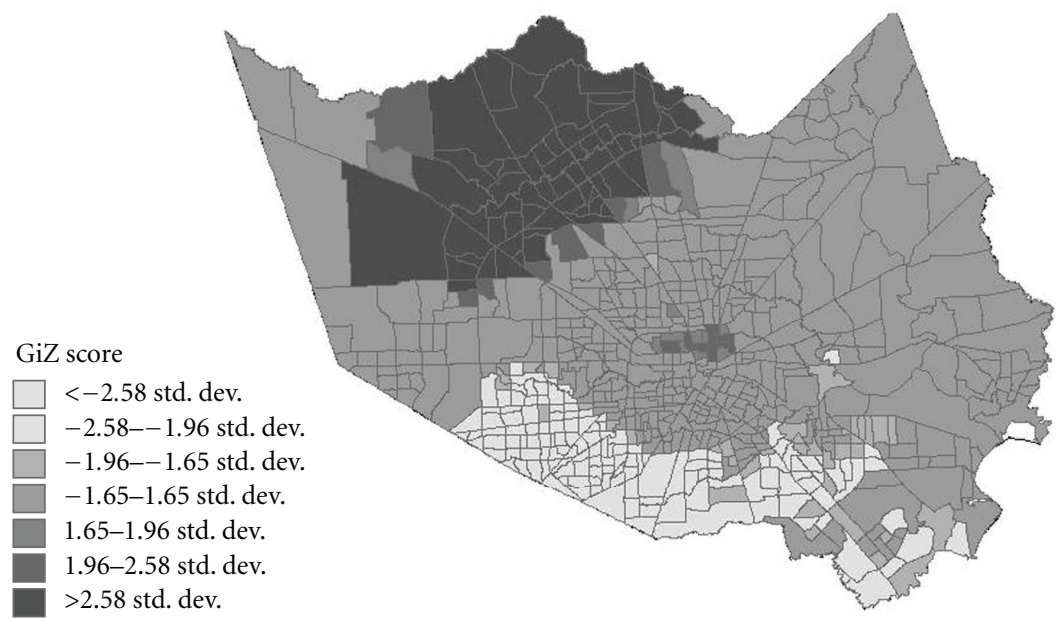

Figure 2: Hot spot cluster analysis of West Nile virus cases in the Houston metropolitan area, Texas.

control method used in this area, it is known that the use of adulticides is random and not associated with specific water bodies. Another speculation is that alternate breeding sites, specifically storm sewers, also play a role in disease transmission. In Houston, Culex quinquefasciatus are the dominate mosquito species collected from storm sewers, and storm sewers have been demonstrated as a preferential site for breeding, larval development, and daytime resting [11]. Unfortunately, we did not have access to sewer blueprints of the metropolitan area to further investigate this theory.

When analyzing residential proximity to water source types, we did find a strongly significant association for risk of human infection among residences near creeks and gullies, specifically Cypress Creek. It is believed that the slower movement of water and dense vegetation is preferential for the local transmitting Culex vector species. Due to low numbers of cases per creek, no additional specific creek sources were included in the final analysis. Cypress Creek is a large water source that flows throughout the northwest corner of the metropolitan Houston area. Figure 1 demonstrates that attack rates of human infection are strongest in the area where Cypress Creek flows. This finding is further substantiated by Figure 2, which shows the highest clusters of human WNV cases are in the area 


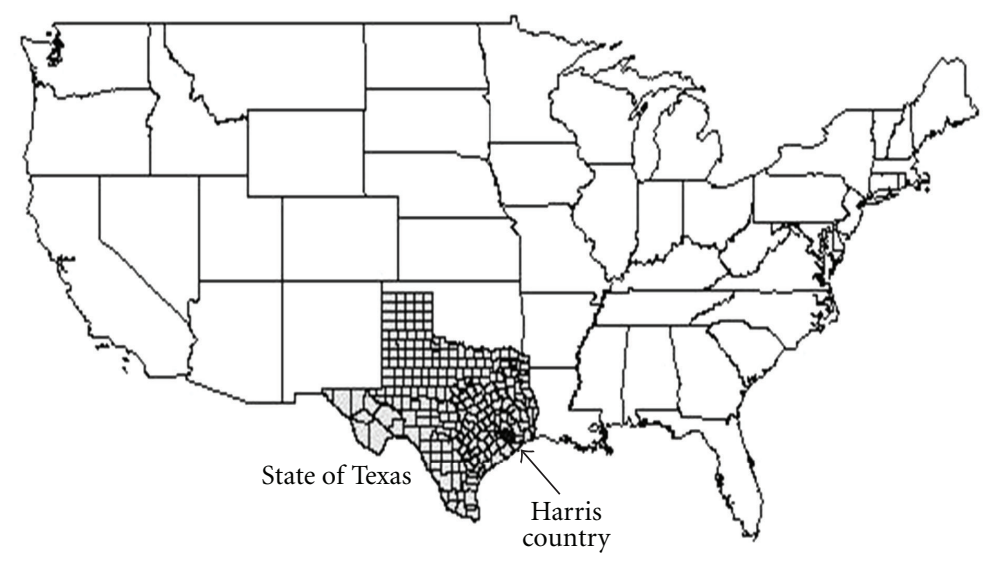

FIGURE 3: Geographic location of metropolitan Houston area inlaid within Harris County in relation to the State of Texas and the United States of America.

where Cypress Creek flows. We feel the true association of infection is with the particular water source Cypress Creek. Additional studies should perform mosquito pool testing around Cypress Creek and additional creeks and gullies throughout the metropolitan area to examine WNV field infection rates of mosquitoes in efforts to further validate our findings.

When analyzing residential proximity to water source types, we did find a strong protective association of residences closest to streams; however, no particular stream water sources were identified as being associated with infection. To further investigate these findings, we created a grouping of moderate moving water sources which included streams and rivers. This grouping had the strongest significance of protection from human WNV infection. Additionally, no particular river water sources were identified as being associated with infection. These findings are evidence that residences in closest proximity to moderate moving water sources are significantly protected against WNV human infection.

Houston is prone to flooding, and as part of the flood mitigation program, the city has an extensive network of bayous, which are man-made canals [2]. The surrounding habitats of bayous in Houston are varied with some being cast with concrete walls and others edged with grass, shrubs, and other vegetation. Overall, we did not find an association between the living near bayous and increased odds of infection. However, we did find that White Oak Bayou and Buffalo Bayou were significantly associated with increased odds of infection. These specific bayous are lined with extensive vegetation preferential to mosquito habitats. This is in sharp contradiction to the bayous lined with concrete, such as Brays Bayou, where the data suggested decreased odds of infection. We cogitate that the type of bayou lining and habitat dictates WNV transmission. Future research should incorporate bayou linings and their individual risk for local human habitants.
There are a few limitations of this study that are worth noting. One limitation was the potential for selection bias due to the inability to verify disease status of controls by serum antibody testing. Since WNV is a mandatoryreportable disease in the state of Texas, anyone who tested positive should have been reported to the local health department. The risk of misclassification of controls is possible if a resident at the address never developed symptoms or had mild disease that went undiagnosed as WNV. However, this risk is presumed minimal since current estimates of seroprevalence in Houston are relatively low [12]. Due to financial constraints, we were unable to obtain a serum sample from controls to verify disease status. Lastly, we were unable to test for potential confounders related to human-mosquito transmission, such as socioeconomic status, gender, rainfall, or other seasonal environmental factors. Complete records for these potential confounders were unavailable. Despite the inability to control for these potential confounds, we believe the results are sound considering people do not choose their residence location based on human-mosquito transmission hotspots.

The main strength of the study is the ability to determine high risk areas of WNV transmission around the Houston metropolitan area using minimal resources. The methods we used are simple to perform and could be of benefit to health authorities in other jurisdictions to identify areas with increased risk for WNV transmission. In resourcescarce public health departments, this inexpensive method could greatly increase the effectiveness of mosquito control programs. Our case-control selection methods would be simple to replicate. Since WNV is a reportable disease nationally, case investigations are performed for all patients that test positive. From these case investigations, health departments should have the addresses of the cases in their jurisdiction. Control selection would be easy to execute as census data is readily available from the US Census Bureau website that is updated both annually and decennially. 
In conclusion, we found that living near slow moving water bodies, such as creeks and gullies, or bayous with heavy vegetation increased one's odds of infection with WNV. Most importantly, we identified Cypress Creek as an area of high WNV human infection that should be targeted by future mosquito control efforts. With the recent literature suggestive of increased ranges of arboviral vectors and areas of transmission, this method of spatial analysis could benefit other health authorities in areas experiencing active WNV transmission who need predictive models of exposure risk for targeted education and control efforts for disease prevention.

\section{Conflict of Interests}

The authors have no conflicts of interests to report.

\section{Acknowledgments}

The authors thank the cohort participants for their contribution to this study, as well as Dr. Keith Bureau, Dr. Irina Cech, Dr. Adebowale Awosika-Olumo, and staff from the City of Houston Department of Health and Human Services, Harris County Public Health and Environmental Services, and Gulf Coast Regional Blood Center for their assistance. This work was funded in part through a grant from the United States Department of Defense, Telemedicine and Advanced Technology Research Center (TexSHIELD W81XWH-07-20031), and the Gillson-Longenbaugh Foundation. This study was approved by the University of Texas Health Science Center at Houston Committee for the Protection of Human Subjects (HSC-SPH-03-039) in collaboration with the other institutions involved in this project.

\section{References}

[1] U S census bureau, 2010, http://www.census.gov/.

[2] K. M. Lillibridge, R. Parsons, Y. Randle et al., "The 2002 introduction of West Nile virus into Harris County, Texas, an area historically endemic for St. Louis encephalitis," American Journal of Tropical Medicine and Hygiene, vol. 70, no. 6, pp. 676-681, 2004.

[3] USGS disease maps, 2010, http://www.diseasemaps.usgs.gov/.

[4] R. B. Tesh, R. Parsons, M. Siirin et al., "Year-round West Nile virus activity, Gulf Coast Region, Texas and Louisiana," Emerging Infectious Diseases, vol. 10, no. 9, pp. 1649-1652, 2004.

[5] R. Matheson, Handbookof the Mosquitoes of North America, Hafner Publishing Company, New York, NY, USA, 2nd edition, 1966.

[6] J. B. Henn, M. E. Metzger, J. A. Kwan et al., "Development time of Culex mosquitoes in stormwater management structures in California," Journal of the American Mosquito Control Association, vol. 24, no. 1, pp. 90-97, 2008.

[7] E. J. Muturi, J. I. Shililu, B. G. Jacob et al., "Diversity of riceland mosquitoes and factors affecting their occurrence and distribution in Mwea, Kenya," Journal of the American Mosquito Control Association, vol. 24, no. 3, pp. 349-358, 2008.

[8] C. M. Barker, B. G. Bolling, C. G. Moore, and L. Eisen, "Relationship between distance from major larval habitats and abundance of adult mosquitoes in semiarid plains landscapes inColorado," Journal of Medical Entomology, vol. 46, no. 6, pp. 1290-1298, 2009.

[9] F. Mostashari, M. L. Bunning, P. T. Kitsutani et al., "Epidemic West Nile encephalitis, New York, 1999: results of a householdbased seroepidemiological survey," The Lancet, vol. 358, no. 9278, pp. 261-264, 2001.

[10] W. K. Reisen, M. M. Milby, and R. P. Meyer, "Population dynamics of adult Culex mosquitoes (diptera: culicidae) along the Kern River, Kern County, California, in 1990," Journal of Medical Entomology, vol. 29, no. 3, pp. 531-543, 1992.

[11] R. Parsons, "Mosquito control-Texas style," Wing Beats, vol. 14, pp. 4-38, 2003.

[12] K. O. Murray, S. Baraniuk, M. Resnick et al., "Risk factors for encephalitis and death from West Nile virus infection," Epidemiology and Infection, vol. 134, no. 6, pp. 1325-1332, 2006. 

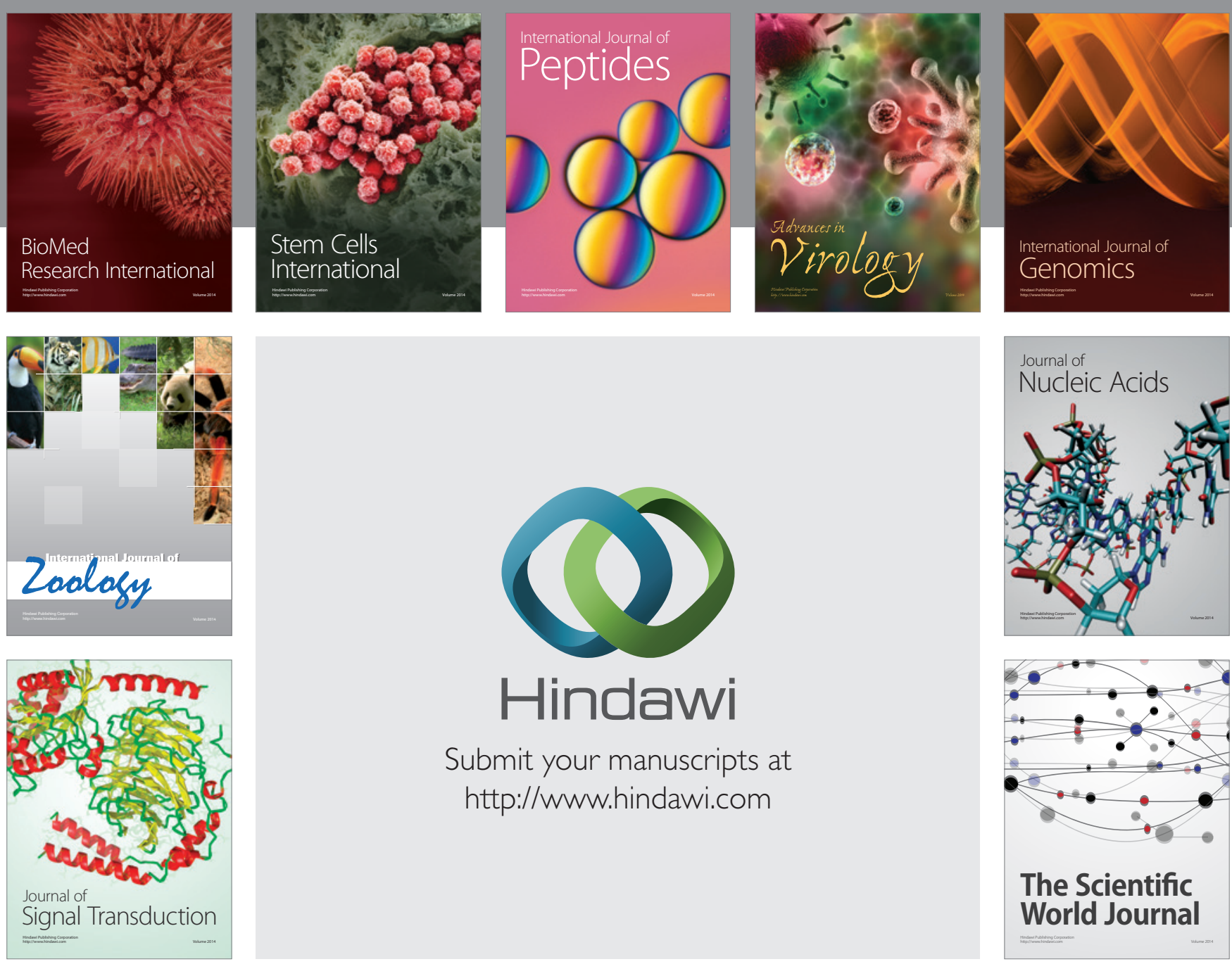

Submit your manuscripts at

http://www.hindawi.com
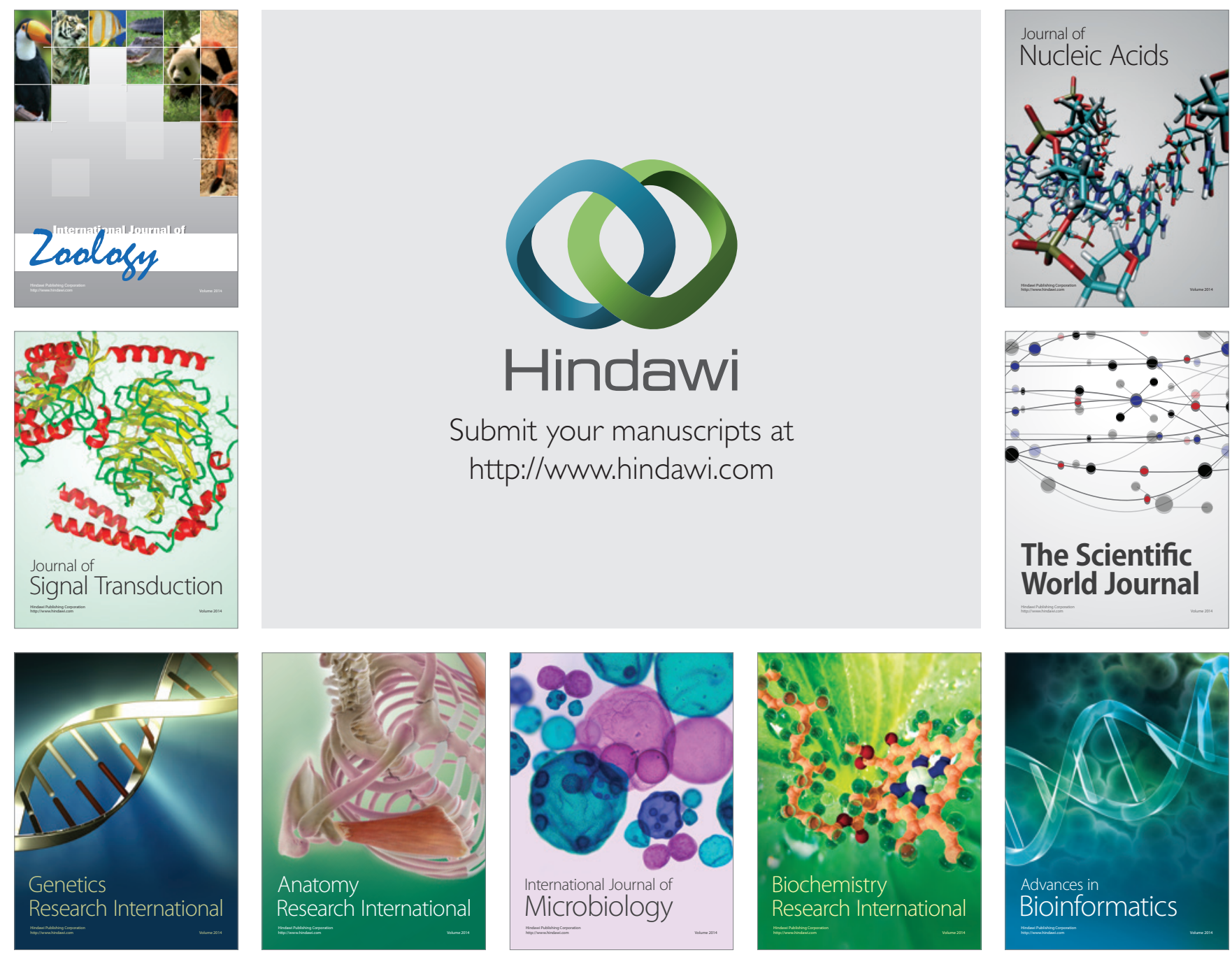

The Scientific World Journal
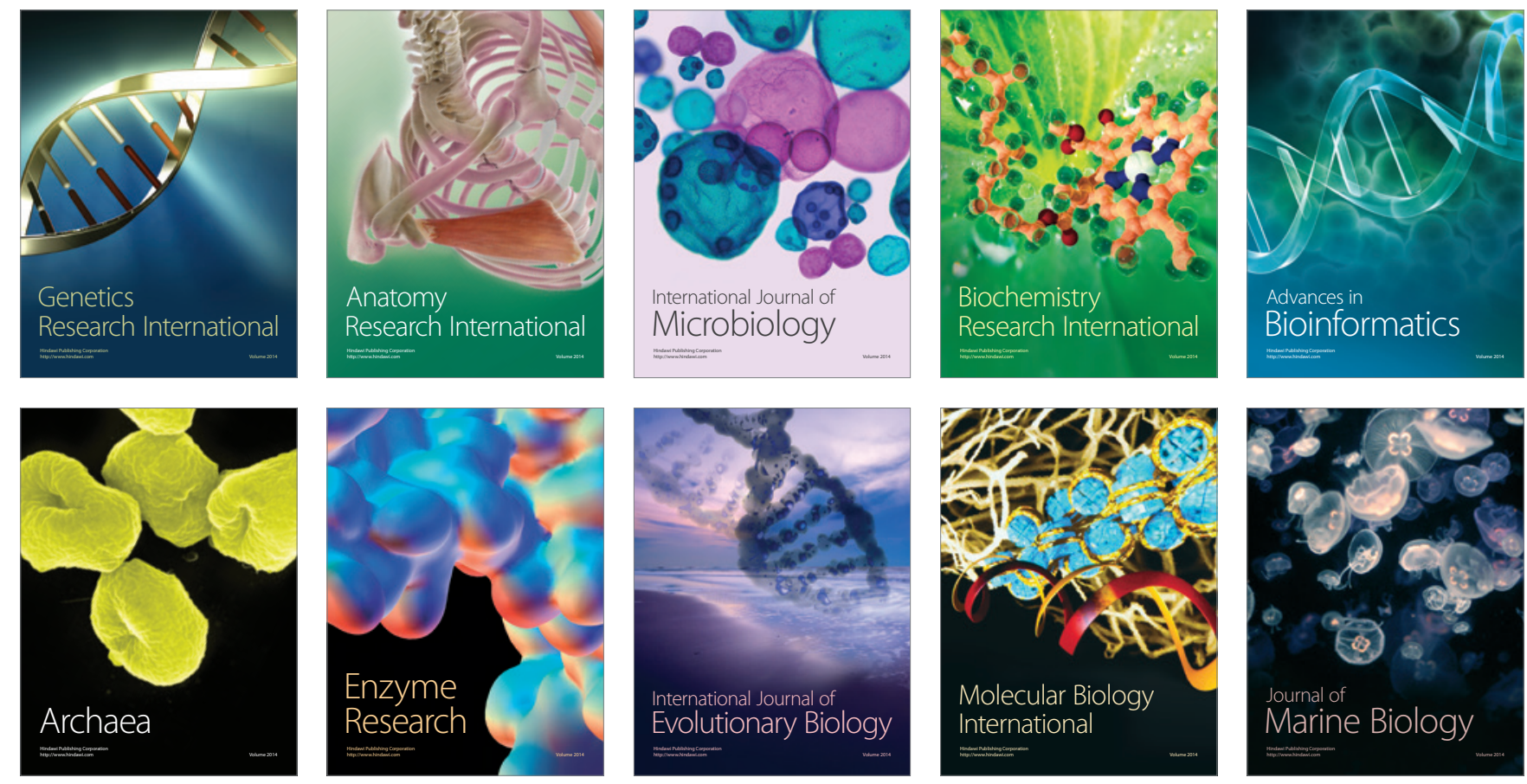